\title{
WEAK TYPE ESTIMATES FOR FUNCTIONS OF MARCINKIEWICZ TYPE WITH FRACTIONAL INTEGRALS OF MIXED HOMOGENEITY
}

\author{
SHUICHI SATO
}

\begin{abstract}
We prove the endpoint weak type estimate for square functions of Marcinkiewicz type with fractional integrals associated with non-isotropic dilations. This generalizes a result of C. Fefferman on functions of Marcinkiewicz type by considering fractional integrals of mixed homogeneity in place of the Riesz potentials of Euclidean structure.
\end{abstract}

\section{INTRODUCTION}

Let $P=\operatorname{diag}\left(a_{1}, \ldots, a_{n}\right)$ be an $n \times n$ real diagonal matrix such that $a_{j} \geq 1$, $1 \leq j \leq n$. Define a dilation group $\left\{A_{t}\right\}_{t>0}$ on $\mathbb{R}^{n}$ by $A_{t}=\operatorname{diag}\left(t^{a_{1}}, \ldots, t^{a_{n}}\right)$. We see that $\left|A_{t} x\right|$ is strictly increasing as a function of $t$ on $\mathbb{R}_{+}=(0, \infty)$ for $x \neq 0$, where $|x|$ denotes the Euclidean norm. Define a norm function $\rho(x), x \neq 0$, to be the unique positive real number $t$ such that $\left|A_{t^{-1}} x\right|=1$ and let $\rho(0)=0$. Then $\rho\left(A_{t} x\right)=t \rho(x), t>0, x \in \mathbb{R}^{n}$, and the following properties of $\rho(x)$ and $A_{t}$ are known (see [2, 4, 9]):

(A) $\rho \in C^{\infty}\left(\mathbb{R}^{n} \backslash\{0\}\right)$;

(B) $\rho(x+y) \leq \rho(x)+\rho(y)$;

(C) $\rho(x) \leq 1$ if and only if $|x| \leq 1$;

(D) $|x| \leq \rho(x)$ if $|x| \leq 1$;

(E) $|x| \geq \rho(x)$ if $|x| \geq 1$;

(F) we have a polar coordinates expression for the Lebesgue measure:

$$
\int_{\mathbb{R}^{n}} f(x) d x=\int_{0}^{\infty} \int_{S^{n-1}} f\left(A_{t} \theta\right) t^{\gamma-1} \mu(\theta) d \sigma(\theta) d t, \quad \gamma=\operatorname{trace} P
$$

where $\mu$ is a strictly positive $C^{\infty}$ function on the unit sphere $S^{n-1}=\{|x|=$ $1\}$ and $d \sigma$ is the Lebesgue surface measure on $S^{n-1}$.

Define a Riesz potential operator by

$$
\widehat{I_{\alpha}(f)}(\xi)=(2 \pi \rho(\xi))^{-\alpha} \hat{f}(\xi)
$$

for $0<\alpha<\gamma$, where the Fourier transform $\hat{f}$ is defined as

$$
\hat{f}(\xi)=\int_{\mathbb{R}^{n}} f(x) e^{-2 \pi i\langle x, \xi\rangle} d x, \quad\langle x, \xi\rangle=\sum_{j=1}^{n} x_{j} \xi_{j},
$$

2010 Mathematics Subject Classification. Primary 42B25; Secondary 46E35.

Key Words and Phrases. Weak type estimates, functions of Marcinkiewicz type, non-isotropic dilations, Riesz potentials.

The author is partly supported by Grant-in-Aid for Scientific Research (C) No. 16K05195, Japan Society for the Promotion of Science. 
with $x=\left(x_{1}, \ldots, x_{n}\right), \xi=\left(\xi_{1}, \ldots, \xi_{n}\right)$ (see also Remark 8.1 in Section 8 for the definition of $\left.I_{\alpha}\right)$. Let $\|f\|_{p}$ denote the $L^{p}$ norm of a function $f$ in $L^{p}\left(\mathbb{R}^{n}\right)$. Let $\mathcal{S}\left(\mathbb{R}^{n}\right)$ be the Schwartz class of rapidly decreasing smooth functions on $\mathbb{R}^{n}$. Then the following result is known (see [3, Theorem 4.1]).

Theorem A. Let $1<p<\infty, 0<\alpha<\gamma / p, 1 / p-1 / q=\alpha / \gamma$. Suppose that $f$ is in $\mathcal{S}\left(\mathbb{R}^{n}\right)$ and $\operatorname{supp}(\hat{f})$ does not contain the origin. Then

$$
\left\|I_{\alpha}(f)\right\|_{q} \leq C\|f\|_{p}
$$

Define

$$
D_{\alpha}(f)(x)=\left(\int_{\mathbb{R}^{n}}\left|I_{\alpha}(f)(x+y)-I_{\alpha}(f)(x)\right|^{2} \rho(y)^{-\gamma-2 \alpha} d y\right)^{1 / 2} .
$$

In this note we shall prove the following.

Theorem 1.1. Let $0<\alpha<1$ and $p_{0}=2 \gamma /(\gamma+2 \alpha)$. Suppose that $p_{0}>1$. Then

(1) the operator $D_{\alpha}$ is bounded on $L^{p}\left(\mathbb{R}^{n}\right)$ if $p_{0}<p<\infty$;

(2) $D_{\alpha}$ is of weak type $\left(p_{0}, p_{0}\right)$ :

$$
\sup _{\beta>0} \beta^{p_{0}}\left|\left\{x \in \mathbb{R}^{n}: D_{\alpha}(f)(x)>\beta\right\}\right| \leq C\|f\|_{p_{0}}^{p_{0}},
$$

where $|E|$ denotes the Lebesgue measure of a set $E$.

We note that $p_{0}>1$ for all $\alpha \in(0,1)$ if $n \geq 2$. See Remark 8.2 in Section 8 for the optimality of Theorem 1.1. When $A_{t} x=t x$ and $\rho(x)=|x|$, part (1) is due to [21] and part (2) is stated in [10, a proof of which can be found in [5. The proof of [5] uses properties of harmonic functions by extending $I_{\alpha}(f)$ as a harmonic function on the upper half space $\mathbb{R}_{+}^{n+1}=\mathbb{R}^{n} \times(0, \infty)$ and results are stated in weighted settings. Also, see [20] for results related to part (1) with $A_{t}=\operatorname{diag}\left(t, \ldots, t, t^{2}\right)$, $\gamma=n+1, n \geq 2$.

In 1938, a square function, now called the Marcinkiewicz function, was introduced by [12] in the setting of periodic functions on $\mathbb{R}^{1}$, which can be used to investigate differentiability of functions and characterize function spaces including Sobolev spaces. A generalization of the Marcinkiewicz function to higher dimensions can be found in [21, where also $D_{\alpha}(f)$, a variant of the Marcinkiewicz function, is considered when $\rho(x)=|x|$. We refer to [1], 11], 15, [16, 17], [18] and [19] for relevant, recent results on the relations between functions of Marcinkiewicz type and Sobolev spaces.

To prove part (1) for $p \in\left(p_{0}, 2\right]$, we first prove $L^{2}$ boundedness of $D_{\alpha}$ by applying the Fourier transform and the result for $p \in\left(p_{0}, 2\right)$ follows from the Marcinkiewicz interpolation theorem between the $L^{2}$ boundedness and the weak type boundedness of part (2).

The proof of part (2) we give in this note is motivated by the proof of the weak type estimate for the Littlewood-Paley function $g_{\lambda}^{*}$ in [10]. The proof of [10] uses some properties of the Poisson kernel

$$
P(x, t)=c_{n} \frac{t}{\left(|x|^{2}+t^{2}\right)^{(n+1) / 2}}, \quad c_{n}=\frac{\Gamma((n+1) / 2)}{\pi^{(n+1) / 2}},
$$


associated with harmonic functions on the upper half space $\mathbb{R}_{+}^{n+1}$ (see 23, Chapter I]). One of them is related to the formula

$$
\int_{0}^{\infty} P_{t} * f(x) t^{\alpha-1} d t=\Gamma(\alpha) I_{\alpha}(f)(x),
$$

where $\widehat{I_{\alpha} f}(\xi)=(2 \pi|\xi|)^{-\alpha} \hat{f}(\xi), P_{t}(x)=t^{-n} P(x / t)=P(x, t)$, with $P(x)=P(x, 1)$. Also, some regularities on $P(x, t)$ are used, although properties of harmonic functions, like that applied in 5 to prove the special case of Theorem 1.1(2) mentioned above, are not used in an essential way. In proving Theorem 1.1 (2), we are able to successfully generalize the methods of [10] for the estimate of $g_{\lambda}^{*}$ to the present situation, where results from differential equations, like harmonicity, are not readily available. Our proof of Theorem 1.1 (2) in this note is new even in the case of the Euclidean norm setting.

To prove part (2) of Theorem 1.1, we consider the function $K$ defined by

$$
K(x)=\int_{\mathbb{R}^{n}} e^{-2 \pi \rho(\xi)} e^{2 \pi i\langle x, \xi\rangle} d \xi,
$$

as a substitute for the Poisson kernel $P(x)$ and consider the function $K_{t} * f(x)$, where $K_{t}(x)=t^{-\gamma} K\left(A_{t}^{-1} x\right)$. Then we have an analogue of (1.3) for the general $I_{\alpha}(f)$ in (1.1) (see (4.3) below). Also, we have some results analogous to the regularities for $P$ (see Lemma 3.1 below). We shall apply these results to estimate the bad part arising from the Calderón-Zygmund decomposition derived from the Whitney type decomposition of open sets in homogeneous spaces (see [6, 7]). To treat the good part we shall apply the $L^{2}$ boundedness of $D_{\alpha}$.

In Section 2, we shall state the Calderón-Zygmund decomposition of $f \in L^{p}\left(\mathbb{R}^{n}\right)$, $1<p<\infty$, at height $\beta^{p}, \beta>0$, needed for the proof of Theorem 1.1 (2). Some properties of functions related to $K$ in (1.4) will be shown in Section 3.

We shall prove the $L^{2}$ boundedness of $D_{\alpha}$ in Section 4. Part (2) of Theorem 1.1 will be shown by applying the $L^{2}$ boundedness and the Calderón-Zygmund decomposition in Sections 4 through 6 . We shall show part (1) of Theorem 1.1 for $p>2$ in Section 7 by proving weighted $L^{2}$ estimates for $D_{\alpha}$ with $A_{1}$-weights. Finally, we shall have some concluding remarks in Section 8.

\section{Decomposition Results}

For $x \in \mathbb{R}^{n}$ and $r>0$, let $B(x, r)$ be the ball centered at $x$ with radius $r$ defined by $\rho: B(x, r)=\left\{y \in \mathbb{R}^{n}: \rho(x-y)<r\right\}$. Then we have the following (see [7] and also [6]).

Lemma 2.1. Let $O$ be an open bounded set in $\mathbb{R}^{n}$ and $N \geq 1$. Then There exists a sequence $\left\{B\left(c_{j}, r_{j}\right)\right\}_{j=1}^{\infty}$ of balls for which we have

(1) $O=\cup_{j=1}^{\infty} B\left(c_{j}, r_{j}\right)$;

(2) there exists $C>0$ such that $\sum_{j=1}^{\infty} \chi_{B\left(c_{j}, N r_{j}\right)} \leq C$, where $\chi_{E}$ denotes the characteristic function of a set $E$;

(3) there exists $C_{1} \geq 1$ such that $B\left(c_{j}, C_{1} N r_{j}\right) \cap\left(\mathbb{R}^{n} \backslash O\right) \neq \emptyset$.

Applying this we can prove the next result (see [6]).

Lemma 2.2. Let $\beta>0, f \in L^{p}, 1 \leq p<\infty$. Suppose that $f$ is compactly supported. Let $N \geq 1$. Then there exists a sequence $\left\{B\left(c_{j}, r_{j}\right)\right\}_{j=1}^{\infty}$ of balls such that 
(1) $\sum_{j} \chi_{B\left(c_{j}, N r_{j}\right)} \leq C$;

(2) $|\Omega| \leq C \beta^{-p}\|f\|_{p}^{p}$, where $\Omega=\cup B\left(c_{j}, r_{j}\right)$;

(3) $|f(x)| \leq C \beta$ if $x \in \mathbb{R}^{n} \backslash \Omega$;

(4) $\left|B\left(c_{j}, r_{j}\right)\right|^{-1} \int_{B\left(c_{j}, r_{j}\right)}|f(x)|^{p} d x \leq C \beta^{p}$.

Proof. Define the Hardy-Littlewood maximal function

$$
M(f)(x)=\sup _{x \in B} \frac{1}{|B|} \int_{B}|f(y)| d y,
$$

where the supremum is taken over all the balls $B$ which contain $x$. Let

$$
\Omega=\left\{x \in \mathbb{R}^{n}: M\left(|f|^{p}\right)>\beta^{p}\right\} .
$$

Then $\Omega$ is open and bounded. Clearly, we have part (3). By Lemma 2.1 with $\Omega$ in place of $O$, we have a sequence $\left\{B\left(c_{j}, r_{j}\right)\right\}_{j=1}^{\infty}$ of balls as in Lemma 2.1. So we have part (1). Also, part (2) holds true since it is known that $M$ is of weak type $(1,1)$.

By part (3) of Lemma 2.1, there exist $h=C_{1} N \geq 1$ and $y \in \mathbb{R}^{n} \backslash \Omega$ such that $y \in B\left(c_{j}, h r_{j}\right)$. Thus

$$
\frac{1}{\left|B\left(c_{j}, r_{j}\right)\right|} \int_{B\left(c_{j}, r_{j}\right)}|f(x)|^{p} d x \leq h^{\gamma} M\left(|f|^{p}\right)(y) \leq h^{\gamma} \beta^{p},
$$

which implies part (4).

Lemma 2.2 is used to prove the following (see [6]).

Lemma 2.3. Let $\beta, f \in L^{p}, p, N$ and $\left\{B\left(c_{j}, r_{j}\right)\right\}_{j=1}^{\infty}$ be as in Lemma 2.2. Then there exist a bounded function $g$ and a sequence $\left\{b_{j}\right\}_{j=1}^{\infty}$ of functions in $L^{p}$ such that

(1) $f=g+\sum_{j=1}^{\infty} b_{j}$;

(2) $|g(x)| \leq C \beta$

(3) $\|g\|_{p} \leq C\|f\|_{p}$;

(4) $b_{j}(x)=0$ if $x \in B\left(c_{j}, r_{j}\right)^{c}$ for all $j$, where $E^{c}$ denotes the complement of a set $E$;

(5) $\int b_{j}(x) d x=0$ for all $j$;

(6) $\left\|b_{j}\right\|_{p}^{p} \leq C \beta^{p}\left|B\left(c_{j}, r_{j}\right)\right|$ for all $j$;

(7) $\sum_{j=1}^{\infty}\left|B\left(c_{j}, r_{j}\right)\right| \leq C \beta^{-p}\|f\|_{p}^{p}$.

Proof. Define a function $h_{j}$ on $\mathbb{R}^{n}$ by

$$
h_{j}(x)=\frac{\chi_{B\left(c_{j}, r_{j}\right)}(x)}{\sum_{j=1}^{\infty} \chi_{B\left(c_{j}, r_{j}\right)}(x)} \quad \text { if } x \in \Omega,
$$

and $h_{j}(x)=0$ if $x \in \Omega^{c}$, where $\Omega=\cup_{j=1}^{\infty} B\left(c_{j}, r_{j}\right)$. Let

$$
g(x)=\sum_{j=1}^{\infty}\left(\frac{1}{\left|B\left(c_{j}, r_{j}\right)\right|} \int_{B\left(c_{j}, r_{j}\right)} f(y) h_{j}(y) d y\right) \chi_{B\left(c_{j}, r_{j}\right)}(x)+f(x) \chi_{\Omega^{c}}(x)
$$

and

$$
b_{j}(x)=f(x) h_{j}(x)-\left(\frac{1}{\left|B\left(c_{j}, r_{j}\right)\right|} \int_{B\left(c_{j}, r_{j}\right)} f(y) h_{j}(y) d y\right) \chi_{B\left(c_{j}, r_{j}\right)}(x) .
$$


Then by the definitions and Lemma 2.2 we easily have the assertions (1) through (6). Also, since $\left\{B\left(c_{j}, r_{j}\right)\right\}_{j=1}^{\infty}$ is finitely overlapping, by part (2) of Lemma 2.2 we have part (7). This completes the proof.

\section{Some estimates for Fourier transforms}

In this section we prove some estimates for the Fourier transform of the function $e^{-2 \pi t \rho(\xi)}$ and its derivatives needed in proving Theorem 1.1.

Lemma 3.1. We have the following estimates:

$$
\begin{gathered}
|K(x)| \leq C(1+\rho(x))^{-\gamma-1}, \quad \text { where } K \text { is as in (1.4) }, \\
|Q(x)| \leq C(1+\rho(x))^{-\gamma-1},
\end{gathered}
$$

where

$$
\begin{gathered}
Q(x)=-\int_{\mathbb{R}^{n}} 2 \pi \rho(\xi) e^{-2 \pi \rho(\xi)} e^{2 \pi i\langle x, \xi\rangle} d \xi, \\
\left|\int_{\mathbb{R}^{n}} \xi_{k} e^{-2 \pi \rho(\xi)} e^{2 \pi i\langle x, \xi\rangle} d \xi\right| \leq C(1+\rho(x))^{-\gamma-1-a_{k}}, \quad 1 \leq k \leq n, \\
\left|\int_{\mathbb{R}^{n}} \xi_{k} \rho(\xi) e^{-2 \pi \rho(\xi)} e^{2 \pi i\langle x, \xi\rangle} d \xi\right| \leq C(1+\rho(x))^{-\gamma-1-a_{k}}, \quad 1 \leq k \leq n, \\
\left|\int_{\mathbb{R}^{n}} \xi_{k} \xi_{l} e^{-2 \pi \rho(\xi)} e^{2 \pi i\langle x, \xi\rangle} d \xi\right| \leq C(1+\rho(x))^{-\gamma-1-a_{k}-a_{l}}, \quad 1 \leq k, l \leq n .
\end{gathered}
$$

To prove this lemma we need the following two estimates for the derivatives of functions involving homogeneous functions (Lemmas 3.2, 3.3).

Lemma 3.2. Let $b=\left(b_{1}, \ldots, b_{n}\right)$ be a multi-index of non-negative integers $b_{j}$, $1 \leq j \leq n$. Let $H$ be homogeneous of degree $m \in \mathbb{R}$ with respect to the dilation $A_{t}$ and in $C^{\infty}\left(\mathbb{R}^{n} \backslash\{0\}\right)$. Then we have

$$
\left|\partial^{b} H(\xi)\right| \leq C_{b} \rho(\xi)^{m-\langle a, b\rangle}, \quad \xi \in \mathbb{R}^{n} \backslash\{0\},
$$

where $\partial^{b}=\partial_{1}^{b_{1}} \ldots \partial_{n}^{b_{n}}$ with $\partial_{j}=\partial / \partial \xi_{j}$ and $a=\left(a_{1}, \ldots, a_{n}\right)$.

Proof. Differentiating in $\xi^{\prime}$ both sides of the equality

$$
t^{m} H\left(\xi^{\prime}\right)=H\left(t^{a_{1}} \xi_{1}^{\prime}, \ldots, t^{a_{n}} \xi_{n}^{\prime}\right), \quad t>0,
$$

which follows from the homogeneity, and putting $t=\rho(\xi), \xi^{\prime}=A_{\rho(\xi)}^{-1} \xi \in S^{n-1}$, we get the estimates as claimed.

Lemma 3.3. Choose $\varphi \in C_{0}^{\infty}$ such that $\varphi(\xi)=1$ if $\rho(\xi) \leq 1 / 2$ and $\operatorname{supp}(\varphi) \subset$ $B(0,1)$. Let $F \in C^{\infty}(\mathbb{R})$. Let $\epsilon_{k}=0$ or $1,1 \leq k \leq n, m \in \mathbb{R}$. Then we have

$$
\left|\partial^{b}\left(\xi_{k}^{\epsilon_{k}} \xi_{l}^{\epsilon_{l}} \rho(\xi)^{m} F(\rho(\xi)) \varphi(\xi)\right)\right| \leq C_{b} \rho(\xi)^{m+\epsilon_{k} a_{k}+\epsilon_{l} a_{l}-\langle a, b\rangle} .
$$

Proof. Applying Lemma 3.2 with $H=\rho$, we observe that

$$
\left|\partial^{b}(F(\rho(\xi)) \varphi(\xi))\right| \leq C_{b} \rho(\xi)^{1-\langle a, b\rangle}, \quad b \neq 0 .
$$

By Leibniz's formula we have

$$
\partial^{b}\left(\xi_{k}^{\epsilon_{k}} \xi_{l}^{\epsilon_{l}} \rho(\xi)^{m} F(\rho(\xi)) \varphi(\xi)\right)=\sum_{b^{\prime}+b^{\prime \prime}=b} C_{b^{\prime}, b^{\prime \prime}}\left[\partial^{b^{\prime}}\left(\xi_{k}^{\epsilon_{k}} \xi_{l}^{\epsilon_{l}} \rho(\xi)^{m}\right)\right]\left[\partial^{b^{\prime \prime}}(F(\rho(\xi)) \varphi(\xi))\right] .
$$


Since $S(\xi)=\xi_{k}^{\epsilon_{k}} \xi_{l}^{\epsilon_{l}} \rho(\xi)^{m}$ is homogeneous of degree $\epsilon_{k} a_{k}+\epsilon_{l} a_{l}+m$, by Lemma 3.2 and (3.6) we see that

$$
\begin{aligned}
& \left|\partial^{b}(S(\xi) F(\rho(\xi)) \varphi(\xi))\right| \leq \sum_{b^{\prime}+b^{\prime \prime}=b} C_{b^{\prime}, b^{\prime \prime}}\left|\partial^{b^{\prime}} S(\xi)\right|\left|\partial^{b^{\prime \prime}}(F(\rho(\xi)) \varphi(\xi))\right| \\
& \leq C \rho(\xi)^{m+\epsilon_{k} a_{k}+\epsilon_{l} a_{l}-\langle a, b\rangle}+C \sum_{b^{\prime}+b^{\prime \prime}=b, b^{\prime \prime} \neq 0} \rho(\xi)^{m+\epsilon_{k} a_{k}+\epsilon_{l} a_{l}-\left\langle a, b^{\prime}\right\rangle} \rho(\xi)^{1-\left\langle a, b^{\prime \prime}\right\rangle},
\end{aligned}
$$

for $\xi \in B(0,1) \backslash\{0\}$. This completes the proof.

Applying Lemma 3.3 and integration by parts, we can prove the following estimate, from which Lemma 3.1 readily follows.

Lemma 3.4. Let $G(\xi)=\xi_{k}^{\epsilon_{k}} \xi_{l}^{\epsilon_{l}} \rho(\xi)^{m} F(\rho(\xi)) \varphi(\xi)$ be as in Lemma 3.3. We assume that $m>-\gamma$. Then

$$
\left|\int_{\mathbb{R}^{n}} G(\xi) e^{2 \pi i\langle x, \xi\rangle} d \xi\right| \leq C(1+\rho(x))^{-\gamma-m-\epsilon_{k} a_{k}-\epsilon_{l} a_{l}} .
$$

Proof. Let $\Phi \in C_{0}^{\infty}\left(\mathbb{R}_{+}\right)$be such that $\operatorname{supp}(\Phi) \subset\{1 / 2 \leq r \leq 2\}, \Phi \geq 0$ and $\sum_{j=0}^{\infty} \Phi\left(2^{j} \rho(\xi)\right)=1$ on $B(0,1) \backslash\{0\}$. Decompose

$$
\int_{\mathbb{R}^{n}} G(\xi) e^{2 \pi i\langle x, \xi\rangle} d \xi=\sum_{j=0}^{\infty} 2^{-j \gamma} \int_{\mathbb{R}^{n}} G\left(A_{2^{-j}} \xi\right) \Phi(\rho(\xi)) e^{2 \pi i\left\langle A_{2^{-j}} x, \xi\right\rangle} d \xi .
$$

We write $x=A_{\rho(x)} x^{\prime}=\left(\rho(x)^{a_{1}} x_{1}^{\prime}, \ldots, \rho(x)^{a_{n}} x_{n}^{\prime}\right)$ with $x^{\prime} \in S^{n-1}$. We may assume that $\left|x_{1}^{\prime}\right|=\max _{1 \leq j \leq n}\left|x_{j}^{\prime}\right|$ without loss of generality. Then applying integration by parts

$$
\begin{aligned}
& 2^{-j \gamma}\left|\int_{\mathbb{R}^{n}} G\left(A_{2^{-j}} \xi\right) \Phi(\rho(\xi)) e^{2 \pi i\left\langle A_{2-j} x, \xi\right\rangle} d \xi\right| \\
& \leq C 2^{-j \gamma} \sum_{h=h^{\prime}+h^{\prime \prime}} \int 2^{-j a_{1} h^{\prime}}\left|\left(\partial_{1}^{h^{\prime}} G\right)\left(A_{2^{-j}} \xi\right)\right|\left|\partial_{1}^{h^{\prime \prime}} \Phi(\rho(\xi))\right|\left(2^{-j a_{1}} \rho(x)^{a_{1}}\right)^{-h} d \xi .
\end{aligned}
$$

By Lemma 3.3 with $b=\left(h^{\prime}, 0, \ldots, 0\right)$ we have

$$
\begin{aligned}
& 2^{-j \gamma}\left|\int_{\mathbb{R}^{n}} G\left(A_{2^{-j}} \xi\right) \Phi(\rho(\xi)) e^{2 \pi i\left\langle A_{2^{-j}} x, \xi\right\rangle} d \xi\right| \\
& \leq C 2^{-j \gamma} \sum_{h=h^{\prime}+h^{\prime \prime}} \int_{1 / 2 \leq \rho(\xi) \leq 2} 2^{-j a_{1} h^{\prime}} 2^{-j\left(m+\epsilon_{k} a_{k}+\epsilon_{l} a_{l}-h^{\prime} a_{1}\right)} 2^{j h a_{1}} \rho(x)^{-h a_{1}} d \xi \\
& \leq C 2^{j\left(h a_{1}-m-\epsilon_{k} a_{k}-\epsilon_{l} a_{l}-\gamma\right)} \rho(x)^{-h a_{1}} \text {. }
\end{aligned}
$$

Thus if $h a_{1}-m-\epsilon_{k} a_{k}-\epsilon_{l} a_{l}-\gamma>0, \rho(x)>1$,

$$
\begin{aligned}
& \sum_{0 \leq j \leq \log _{2} \rho(x)} 2^{-j \gamma}\left|\int_{\mathbb{R}^{n}} G\left(A_{2^{-j}} \xi\right) \Phi(\rho(\xi)) e^{2 \pi i\left\langle A_{2^{-j}} x, \xi\right\rangle} d \xi\right| \\
& \leq C \rho(x)^{-h a_{1}} \sum_{0 \leq j \leq \log _{2} \rho(x)} 2^{j\left(h a_{1}-m-\epsilon_{k} a_{k}-\epsilon_{l} a_{l}-\gamma\right)} \leq C \rho(x)^{-m-\epsilon_{k} a_{k}-\epsilon_{l} a_{l}-\gamma} .
\end{aligned}
$$


Also, by Lemma 3.3 with $b=0$ we see that

$$
\begin{aligned}
\sum_{j>\log _{2} \rho(x)} 2^{-j \gamma} & \left|\int_{\mathbb{R}^{n}} G\left(A_{2^{-j}} \xi\right) \Phi(\rho(\xi)) e^{2 \pi i\left\langle A_{2^{-j}} x, \xi\right\rangle} d \xi\right| \\
& \leq C \sum_{j>\log _{2} \rho(x)} 2^{j\left(-m-\epsilon_{k} a_{k}-\epsilon_{l} a_{l}-\gamma\right)} \leq C \rho(x)^{-m-\epsilon_{k} a_{k}-\epsilon_{l} a_{l}-\gamma} .
\end{aligned}
$$

Combining (3.7) and (3.8), we get the desired result, since the estimate for $\rho(x) \leq 1$ is obvious.

Proof of Lemma 3.1. Let $\varphi$ be as in Lemma 3.3. Decompose

$$
e^{-2 \pi \rho(\xi)}=-2 \pi \rho(\xi) A(-2 \pi \rho(\xi)) \varphi(\xi)+\varphi(\xi)+e^{-2 \pi \rho(\xi)}(1-\varphi(\xi)),
$$

where $A(s)=\left(e^{s}-1\right) / s$. To prove (3.1), it suffice to show that

$$
\left|\int_{\mathbb{R}^{n}} \rho(\xi) A(-2 \pi \rho(\xi)) \varphi(\xi) e^{2 \pi i\langle x, \xi\rangle} d \xi\right| \leq C(1+\rho(x))^{-\gamma-1},
$$

which follows from Lemma 3.4 with $m=1, \epsilon_{k}=0, \epsilon_{l}=0$. The other estimates can be shown similarly by applying Lemma 3.4 suitably.

\section{Outline of Proof of Theorem 1.1 for $p \in\left[p_{0}, 2\right]$}

We first prove $L^{2}$ boundedness of $D_{\alpha}$ for $0<\alpha<1$. By the Plancherel theorem we have

$$
\begin{aligned}
\left\|D_{\alpha}(f)\right\|_{2}^{2} & =\int_{\mathbb{R}^{n}} \rho(y)^{-\gamma-2 \alpha}\left(\int_{\mathbb{R}^{n}}\left|I_{\alpha}(f)(x+y)-I_{\alpha}(f)(x)\right|^{2} d x\right) d y \\
& =\int_{\mathbb{R}^{n}} \rho(y)^{-\gamma-2 \alpha}\left(\int_{\mathbb{R}^{n}}\left|(2 \pi \rho(\xi))^{-\alpha} \hat{f}(\xi)\left(e^{2 \pi i\langle y, \xi\rangle}-1\right)\right|^{2} d \xi\right) d y \\
& =(2 \pi)^{-2 \alpha} \int_{\mathbb{R}^{n}}|\hat{f}(\xi)|^{2}\left(\int_{\mathbb{R}^{n}}\left|e^{2 \pi i\left\langle y, \xi^{\prime}\right\rangle}-1\right|^{2} \rho(y)^{-\gamma-2 \alpha} d y\right) d \xi,
\end{aligned}
$$

where $\xi^{\prime}=A_{\rho(\xi)}^{-1} \xi$. By (D) of Section 1 we have

$$
\int_{\rho(y) \leq 1}\left|e^{2 \pi i\left\langle y, \xi^{\prime}\right\rangle}-1\right|^{2} \rho(y)^{-\gamma-2 \alpha} d y \leq \int_{\rho(y) \leq 1} 4 \pi^{2} \rho(y)^{2} \rho(y)^{-\gamma-2 \alpha} d y<\infty
$$

since $\alpha<1$. Also, we have

$$
\int_{\rho(y) \geq 1}\left|e^{2 \pi i\left\langle y, \xi^{\prime}\right\rangle}-1\right|^{2} \rho(y)^{-\gamma-2 \alpha} d y \leq \int_{\rho(y) \geq 1} 4 \rho(y)^{-\gamma-2 \alpha} d y<\infty
$$

for $\alpha>0$. Combining results, we see that

$$
\left\|D_{\alpha}(f)\right\|_{2}^{2} \leq C\|\hat{f}\|_{2}^{2}=C\|f\|_{2}^{2},
$$

which proves the $L^{2}$ boundedness.

To prove (1.2) we may assume that $f$ is bounded and compactly supported. Let $\beta>0, p_{0}=2 \gamma /(\gamma+2 \alpha)$. We apply Lemmas 2.2 and 2.3 with these $f, \beta$ and with $N=2, p=p_{0}$. Then we have the sequence $\left\{B\left(c_{j}, r_{j}\right)\right\}_{j=1}^{\infty}$ of balls of Lemma 2.2 and the decomposition $f=g+b, b=\sum_{j=1}^{\infty} b_{j}$, of Lemma 2.3. It suffices to prove

$$
\left|\left\{x \in \mathbb{R}^{n}: D_{\alpha}(g)(x)>\beta\right\}\right| \leq C \beta^{-p_{0}}\|f\|_{p_{0}}^{p_{0}}
$$


and

$$
\left|\left\{x \in \mathbb{R}^{n}: D_{\alpha}(b)(x)>\beta\right\}\right| \leq C \beta^{-p_{0}}\|f\|_{p_{0}}^{p_{0}} .
$$

The estimate (4.1) easily follows from the $L^{2}$ boundedness of $D_{\alpha}$ as follows. By Chebyshev's inequality along with (2) and (3) of Lemma 2.3, since $1<p_{0}<2$, we have

$$
\begin{aligned}
\mid\left\{x \in \mathbb{R}^{n}: D_{\alpha}(g)\right. & (x)>\beta\} \mid \\
\leq & \beta^{-2}\left\|D_{\alpha}(g)\right\|_{2}^{2} \leq C \beta^{-2}\|g\|_{2}^{2} \leq C \beta^{-p_{0}}\|g\|_{p_{0}}^{p_{0}} \leq C \beta^{-p_{0}}\|f\|_{p_{0}}^{p_{0}} .
\end{aligned}
$$

It remains to prove (4.2). Let $K$ be as in (1.4) and

$$
v(x, t)=K_{t} * b(x), \quad V(x, t)=K_{t} * I_{\alpha}(b)(x) .
$$

Then

$$
V(x, t)=\frac{1}{\Gamma(\alpha)} \int_{0}^{\infty} v(x, t+s) s^{\alpha-1} d s .
$$

We have

$$
\begin{aligned}
\mid I_{\alpha}(b)(x+y)- & I_{\alpha}(b)(x)|\leq| V(x+y, \rho(y))-I_{\alpha}(b)(x+y) \mid \\
& +\left|V(x, \rho(y))-I_{\alpha}(b)(x)\right|+|V(x+y, \rho(y))-V(x, \rho(y))| .
\end{aligned}
$$

Let

$$
\begin{aligned}
& J^{(1)}(x)=\Gamma(\alpha)^{2} \int_{\mathbb{R}^{n}}\left|V(y, \rho(y-x))-I_{\alpha}(b)(y)\right|^{2} \rho(y-x)^{-\gamma-2 \alpha} d y, \\
& J^{(2)}(x)=\Gamma(\alpha)^{2} \int_{\mathbb{R}^{n}}\left|V(x, \rho(y-x))-I_{\alpha}(b)(x)\right|^{2} \rho(y-x)^{-\gamma-2 \alpha} d y, \\
& J^{(3)}(x)=\Gamma(\alpha)^{2} \int_{\mathbb{R}^{n}}|V(y, \rho(y-x))-V(x, \rho(y-x))|^{2} \rho(y-x)^{-\gamma-2 \alpha} d y .
\end{aligned}
$$

By (4.3) we can rewrite

$$
\begin{aligned}
& J^{(1)}(x)=\int_{\mathbb{R}^{n}}\left|\int_{0}^{\infty} d t \int_{0}^{\rho(y-x)} \partial_{0} v(y, s+t) t^{\alpha-1} d s\right|^{2} \rho(y-x)^{-\gamma-2 \alpha} d y, \\
& J^{(2)}(x)=\int_{\mathbb{R}^{n}}\left|\int_{0}^{\infty} d t \int_{0}^{\rho(y-x)} \partial_{0} v(x, s+t) t^{\alpha-1} d s\right|^{2} \rho(y-x)^{-\gamma-2 \alpha} d y,
\end{aligned}
$$

where $\partial_{0}=\partial / \partial s$. Let $\Omega_{1}=\cup_{j} B\left(c_{j}, 2 r_{j}\right), \Omega_{2}=\cup_{j} B\left(c_{j}, 4 r_{j}\right)$. Since $\left|\Omega_{2}\right| \leq$ $C \beta^{-p_{0}}\|f\|_{p_{0}}^{p_{0}}$, by (4.4), the estimate (4.2) follows from the inequalities

$$
\begin{aligned}
& \int_{\Omega_{2}^{c}} J^{(1)}(x) d x \leq C \beta^{2-p_{0}}\|f\|_{p_{0}}^{p_{0}}, \\
& \int_{\Omega_{2}^{c}} J^{(2)}(x) d x \leq C \beta^{2-p_{0}}\|f\|_{p_{0}}^{p_{0}}, \\
& \int_{\Omega_{2}^{c}} J^{(3)}(x) d x \leq C \beta^{2-p_{0}}\|f\|_{p_{0}}^{p_{0}} .
\end{aligned}
$$

This will prove part (2) of Theorem 1.1] As mentioned in Section 1, part (1) for $p \in\left(p_{0}, 2\right)$ follows by the Marcinkiewicz interpolation theorem between the estimate in part (2) and the $L^{2}$ boundedness. This will complete the proof of Theorem 1.1 for $p \in\left[p_{0}, 2\right]$. 
We shall prove (4.5), (4.6) in Section 5 and (4.7) in Section 6.

\section{Proofs of the estimates (4.5) and (4.6)}

We first prove the estimate (4.5). Let $\chi(r)=\chi_{(0.1]}(r)$. Then

$$
J^{(1)}(x)=\int_{\mathbb{R}^{n}}\left|\int_{0}^{\infty} \int_{0}^{\infty} \chi\left(\frac{s}{\rho(y-x)}\right) \chi\left(\frac{s}{t}\right)\right| t-\left.\left.s\right|^{\alpha-1} \partial_{0} v(y, t) d t d s\right|^{2} \rho(y-x)^{-\gamma-2 \alpha} d y .
$$

We have

$$
\int_{0}^{t \wedge \rho(y-x)}(t-s)^{\alpha-1} d s=W_{\alpha}(t, \rho(y-x))
$$

where

$$
W_{\alpha}(t, s)=\frac{1}{\alpha}\left(t^{\alpha}-(t-(t \wedge s))^{\alpha}\right), \quad t, s \geq 0,
$$

with $a \wedge b=\min (a, b)$. We note that $W_{\alpha} \geq 0$ and $\int_{0}^{\infty} W_{\alpha}(t, 1) d t / t<\infty$ when $0<\alpha<1$. Using (5.1), we write

$$
J^{(1)}(x)=\int_{\mathbb{R}^{n}}\left|\int_{0}^{\infty} W_{\alpha}(t, \rho(y-x)) \partial_{0} v(y, t) d t\right|^{2} \rho(y-x)^{-\gamma-2 \alpha} d y .
$$

Define $v_{j}(y, t)=b_{j} * K_{t}(y)$ and $B_{j}=B\left(c_{j}, r_{j}\right), \widetilde{B}_{j}=B\left(c_{j}, 2 r_{j}\right)$. Let

$$
J_{1}^{(1)}(x)=\int_{\mathbb{R}^{n}}\left|\int_{0}^{\infty} W_{\alpha}(t, \rho(y-x)) \sum_{y \in \widetilde{B}_{j}^{c}} \partial_{0} v_{j}(y, t) d t\right|^{2} \rho(y-x)^{-\gamma-2 \alpha} d y,
$$

where $\sum_{y \in \widetilde{B}_{j}^{c}}$ means that the summation is over all $j$ such that $y \in \widetilde{B}_{j}^{c}$; similar notation will be used in what follows, and let

$$
J_{2}^{(1)}(x)=\Gamma(\alpha)^{2} \int_{\mathbb{R}^{n}}\left|\sum_{y \in \widetilde{B}_{j}}\left(K_{\rho(y-x)} * I_{\alpha}\left(b_{j}\right)(y)-I_{\alpha}\left(b_{j}\right)(y)\right)\right|^{2} \rho(y-x)^{-\gamma-2 \alpha} d y
$$

To estimate $J_{1}^{(1)}$, we show that

$$
\left|\sum_{y \in \widetilde{B}_{j}^{c}} \partial_{0} v_{j}(y, t)\right| \leq C \beta / t
$$

This can be seen as follows. Let $E(x)=(1+\rho(x))^{-\gamma-1}$. Note that if $y \in \widetilde{B}_{j}^{c}$

$$
\sup _{z \in B_{j}} E_{t}(y-z) \leq C \inf _{z \in B_{j}} E_{t}(y-z) .
$$


Therefore, by Lemma 2.2 (1), Lemma 2.3 and (3.2),

$$
\begin{aligned}
\left|\sum_{y \in \widetilde{B}_{j}^{c}} \partial_{0} v_{j}(y, t)\right| & \leq C t^{-1} \sum_{y \in \widetilde{B}_{j}^{c}} \sup _{z \in B_{j}} E_{t}(y-z) \int\left|b_{j}(z)\right| d z \\
& \leq C t^{-1} \sum_{y \in \widetilde{B}_{j}^{c}}\left(\sup _{z \in B_{j}} E_{t}(y-z)\right) \beta\left|B_{j}\right| \\
& \leq C t^{-1} \beta \sum_{y \in \widetilde{B}_{j}^{c}} \int \chi_{B_{j}}(z) E_{t}(y-z) d z \\
& \leq C t^{-1} \beta\|E\|_{1} .
\end{aligned}
$$

Thus

$$
\begin{aligned}
\left|\int_{0}^{\infty} W_{\alpha}(t, \rho(y-x)) \sum_{y \in \widetilde{B}_{j}^{c}} \partial_{0} v_{j}(y, t) d t\right| & \leq C \beta \int_{0}^{\infty} W_{\alpha}(t, \rho(y-x)) \frac{d t}{t} \\
& =C \beta \rho(y-x)^{\alpha} \int_{0}^{\infty} W_{\alpha}(t, 1) \frac{d t}{t},
\end{aligned}
$$

and hence

$$
\int_{\mathbb{R}^{n}} J_{1}^{(1)}(x) d x \leq C \beta \int_{\mathbb{R}^{n}} \int_{0}^{\infty}\left(\int_{\mathbb{R}^{n}} W_{\alpha}(t, \rho(x)) \rho(x)^{-\gamma-\alpha} d x\right)\left|\sum_{y \in \widetilde{B}_{j}^{c}} \partial_{0} v_{j}(y, t)\right| d y d t .
$$

We note that

$$
\int_{\mathbb{R}^{n}} W_{\alpha}(t, \rho(x)) \rho(x)^{-\gamma-\alpha} d x=\int_{\mathbb{R}^{n}} W_{\alpha}(1, \rho(x)) \rho(x)^{-\gamma-\alpha} d x<\infty,
$$

if $0<\alpha<1$. This implies that

$$
\int_{\mathbb{R}^{n}} J_{1}^{(1)}(x) d x \leq C \beta \iint_{\mathbb{R}_{+}^{n+1}}\left|\sum_{y \in \widetilde{B}_{j}^{c}} \partial_{0} v_{j}(y, t)\right| d y d t
$$

Since $\int b_{j}=0$, if $y \in \widetilde{B}_{j}^{c}$, by (3.4) and Taylor's formula we see that

$$
\begin{aligned}
\left|\partial_{0} v_{j}(y, t)\right| & =t^{-1}\left|Q_{t} * b_{j}(y)\right|=t^{-1}\left|\int_{B_{j}}\left(Q_{t}(y-z)-Q_{t}\left(y-c_{j}\right)\right) b_{j}(z) d z\right| \\
& \leq C \sum_{k=1}^{n} t^{-1-a_{k}-\gamma}\left(1+t^{-1} \rho\left(y-c_{j}\right)\right)^{-\gamma-1-a_{k}} \int_{B_{j}}\left|\left(z-c_{j}\right)_{k}\right|\left|b_{j}(z)\right| d z \\
& \leq C \sum_{k=1}^{n} r_{j}^{a_{k}} t^{-1-a_{k}-\gamma}\left(1+t^{-1} \rho\left(y-c_{j}\right)\right)^{-\gamma-1-a_{k}} \int_{B_{j}}\left|b_{j}(z)\right| d z \\
& \leq C \beta \sum_{k=1}^{n} r_{j}^{a_{k}} t^{-1-a_{k}-\gamma}\left(1+t^{-1}\left(\rho\left(y-c_{j}\right)+r_{j}\right)\right)^{-\gamma-1-a_{k}}\left|B_{j}\right|
\end{aligned}
$$


where the penultimate inequality follows from the estimate $\left|x_{k}\right| \leq C \rho(x)^{a_{k}}$. Therefore, $\int_{\mathbb{R}^{n}} J_{1}^{(1)}(x) d x$ is bounded by

$$
C \beta^{2} \sum_{j}\left|B_{j}\right| \sum_{k=1}^{n} \iint_{\mathbb{R}_{+}^{n+1}} r_{j}^{a_{k}} t^{-1-a_{k}-\gamma}\left(1+t^{-1}\left(\rho\left(y-c_{j}\right)+r_{j}\right)\right)^{-\gamma-1-a_{k}} d y d t .
$$

It is easy to see that the last integral is equal to

$$
\iint_{\mathbb{R}_{+}^{n+1}}(1+t)^{-\gamma-1-a_{k}}(\rho(y)+1)^{-\gamma-a_{k}} d y d t .
$$

Thus, by Lemma 2.3 (7) with $p=p_{0}$, we have

$$
\int_{\mathbb{R}^{n}} J_{1}^{(1)}(x) d x \leq C \beta^{2} \sum_{j}\left|B_{j}\right| \leq C \beta^{2-p_{0}} \mid\|f\|_{p_{0}}^{p_{0}}
$$

Next, we evaluate $J_{2}^{(1)}$. By Schwarz's inequality and Lemma 2.2 (1), we see that

$$
\begin{aligned}
J_{2}^{(1)}(x) & \leq C \int_{\Omega_{1}}\left|\sum_{j} M\left(I_{\alpha} b_{j}\right)(y) \chi_{B\left(c_{j}, 2 r_{j}\right)}(y)\right|^{2} \rho(y-x)^{-\gamma-2 \alpha} d y \\
& \leq C \sum_{j} \int_{B\left(c_{j}, 2 r_{j}\right)}\left|M\left(I_{\alpha} b_{j}\right)(y)\right|^{2} \rho(y-x)^{-\gamma-2 \alpha} d y .
\end{aligned}
$$

Let $x \in \Omega_{2}^{c}$. Then by the $L^{2}$ boundedness of the maximal operator $M$ we have

$$
\begin{aligned}
J_{2}^{(1)}(x) & \leq C \sum_{j} \rho\left(x-c_{j}\right)^{-\gamma-2 \alpha} \int_{B\left(c_{j}, 2 r_{j}\right)}\left|M\left(I_{\alpha} b_{j}\right)(y)\right|^{2} d y \\
& \leq C \sum_{j} \rho\left(x-c_{j}\right)^{-\gamma-2 \alpha}\left\|I_{\alpha} b_{j}\right\|_{2}^{2} .
\end{aligned}
$$

So, from Theorem A and Lemma 2.3 (6) with $p=p_{0}$ it follows that

$$
J_{2}^{(1)}(x) \leq C \sum_{j} \rho\left(x-c_{j}\right)^{-\gamma-2 \alpha}\left\|b_{j}\right\|_{p_{0}}^{2} \leq C \sum_{j} \rho\left(x-c_{j}\right)^{-\gamma-2 \alpha} \beta^{2}\left|B_{j}\right|^{2 / p_{0}} .
$$

Consequently,

$$
\begin{aligned}
\int_{\Omega_{2}^{c}} J_{2}^{(1)}(x) d x & \leq C \beta^{2} \sum_{j}\left|B_{j}\right|^{2 / p_{0}} \int_{\rho\left(x-c_{j}\right) \geq 4 r_{j}} \rho\left(x-c_{j}\right)^{-\gamma-2 \alpha} d x \\
& \leq C \beta^{2} \sum_{j}\left|B_{j}\right|^{2 / p_{0}} r_{j}^{-2 \alpha} \\
& \leq C \beta^{2} \sum_{j}\left|B_{j}\right|,
\end{aligned}
$$

and hence Lemma 2.3 (7) implies

$$
\int_{\Omega_{2}^{c}} J_{2}^{(1)}(x) d x \leq C \beta^{2-p_{0}}\|f\|_{p_{0}}^{p_{0}} .
$$

The estimate (4.5) follows from (5.2) and (5.3), since $J^{(1)} \leq 2 J_{1}^{(1)}+2 J_{2}^{(1)}$. 
SHUICHI SATO

Let us prove the estimate (4.6) next. In the same way as in the case of $J^{(1)}$, we can write

$$
J^{(2)}(x)=\int_{\mathbb{R}^{n}}\left|\int_{0}^{\infty} W_{\alpha}(t, \rho(y-x)) \partial_{0} K_{t} * b(x) d t\right|^{2} \rho(y-x)^{-\gamma-2 \alpha} d y .
$$

Interchanging the order of integration on the left hand side of (4.6), we have

$$
\begin{aligned}
\int_{\Omega_{2}^{c}} J^{(2)} & (x) d x \\
& =\int_{\mathbb{R}^{n}}\left(\int_{\Omega_{2}^{c}}\left|\int_{0}^{\infty} W_{\alpha}(t, \rho(y-x)) \partial_{0} v(x, t) d t\right|^{2} \rho(y-x)^{-\gamma-2 \alpha} d x\right) d y .
\end{aligned}
$$

For $x \in \Omega_{2}^{c}$, we note that $\partial_{0} v(x, t)=\sum_{x \in \widetilde{B}_{j}^{c}} \partial_{0} v_{j}(x, t)$. Thus we can prove (4.6) in the same way as (5.2).

\section{Proof of the estimate (4.7)}

We note that

$V(x+y, \rho(y))-V(x, \rho(y))=\frac{1}{\Gamma(\alpha)} \int_{0}^{\infty}\left(f * K_{t+\rho(y)}(x+y)-f * K_{t+\rho(y)}(x)\right) t^{\alpha-1} d t$.

Thus we have

$$
\begin{aligned}
& J^{(3)}(x) \\
= & \int_{\mathbb{R}^{n}}\left|\int_{0}^{\infty}\left(b * K_{t}(y)-b * K_{t}(x)\right)\right| t-\left.\left.\rho(y-x)\right|^{\alpha-1} \chi\left(\frac{\rho(y-x)}{t}\right) d t\right|^{2} \rho(y-x)^{-\gamma-2 \alpha} d y .
\end{aligned}
$$

Let

$$
A_{j}(x, y)=\int_{0}^{\infty}\left(\int\left(K_{t}(y-z)-K_{t}(x-z)\right) b_{j}(z) d z\right)|t-\rho(y-x)|^{\alpha-1} \chi\left(\frac{\rho(y-x)}{t}\right) d t,
$$

with $y \in \widetilde{B}_{j}^{c}$. By (3.3) and Taylor's formula, if $z \in B_{j}$ and $\rho(x-y)<t$ we have

$$
\begin{aligned}
\left|K_{t}(y-z)-K_{t}(x-z)\right| & \leq C \sum_{k=1}^{n}\left|y_{k}-x_{k}\right| t^{-a_{k}-\gamma}\left(1+t^{-1} \rho\left(y-c_{j}\right)\right)^{-\gamma-1-a_{k}} \\
& \leq C \sum_{k=1}^{n} \rho(y-x)^{a_{k}} t^{-a_{k}-\gamma} \inf _{z \in B_{j}}\left(1+t^{-1} \rho(y-z)\right)^{-\gamma-1-a_{k}} .
\end{aligned}
$$

Therefore if $\rho(x-y)<t$, by Lemma $2.3(6)$,

$$
\begin{aligned}
\mid \int\left(K_{t}(y-z)-\right. & \left.K_{t}(x-z)\right) b_{j}(z) d z \mid \\
\leq & C \beta \sum_{k=1}^{n} \rho(y-x)^{a_{k}} t^{-a_{k}-\gamma} \int_{B_{j}}\left(1+t^{-1} \rho(y-z)\right)^{-\gamma-1-a_{k}} d z,
\end{aligned}
$$

and hence Lemma 2.2 (1) implies that

$$
\sum_{y \in \widetilde{B}_{j}^{c}}\left|\int\left(K_{t}(y-z)-K_{t}(x-z)\right) b_{j}(z) d z\right| \leq C \beta \sum_{k=1}^{n} C_{k} \rho(y-x)^{a_{k}} t^{-a_{k}}
$$


with $C_{k}=\int_{\mathbb{R}^{n}}(1+\rho(z))^{-\gamma-1-a_{k}} d z$. Consequently,

$$
\begin{aligned}
\sum_{y \in \widetilde{B}_{j}^{c}}\left|A_{j}(x, y)\right| & =C \beta \sum_{k=1}^{n} C_{k} \rho(y-x)^{a_{k}} \int_{\rho(y-x)}^{\infty} t^{-a_{k}}(t-\rho(y-x))^{\alpha-1} d t \\
& =C \beta \rho(y-x)^{\alpha} \sum_{k=1}^{n}\left(C_{k} \int_{1}^{\infty} t^{-a_{k}}(t-1)^{\alpha-1} d t\right) .
\end{aligned}
$$

Thus we have

$$
\sum_{y \in \widetilde{B}_{j}^{c}}\left|A_{j}(x, y)\right| \leq C \beta \rho(y-x)^{\alpha}
$$

Let $J_{1}^{(3)}(x)$ be

$$
\int_{\mathbb{R}^{n}}\left|\int_{0}^{\infty} \sum_{y \in \widetilde{B}_{j}^{c}}\left(v_{j}(y, t)-v_{j}(x, t)\right)\right| t-\left.\left.\rho(y-x)\right|^{\alpha-1} \chi\left(\frac{\rho(y-x)}{t}\right) d t\right|^{2} \rho(y-x)^{-\gamma-2 \alpha} d y
$$

and let

$$
J_{2}^{(3)}(x)=\Gamma(\alpha)^{2} \int_{\mathbb{R}^{n}}\left|\sum_{y \in \widetilde{B}_{j}}\left(K_{\rho(x-y)} * I_{\alpha}\left(b_{j}\right)(y)-K_{\rho(x-y)} * I_{\alpha}\left(b_{j}\right)(x)\right)\right|^{2} \rho(x-y)^{-\gamma-2 \alpha} d y .
$$

Then $J^{(3)} \leq 2 J_{1}^{(3)}+2 J_{2}^{(3)}$. From (6.1) we see that $J_{1}^{(3)}(x)$ is majorized by

$C \beta \int_{\mathbb{R}^{n}}\left|\int_{0}^{\infty} \sum_{y \in \widetilde{B}_{j}^{c}}\left(v_{j}(y, t)-v_{j}(x, t)\right)\right| t-\left.\rho(y-x)\right|^{\alpha-1} \chi\left(\frac{\rho(y-x)}{t}\right) d t \mid \rho(y-x)^{-\gamma-\alpha} d y$.

Let

$$
R(t, y, x, z)=K_{t}(y-z)-K_{t}(x-z) .
$$

Then

$v_{j}(y, t)-v_{j}(x, t)=\int R(t, y, x, z) b_{j}(z) d z=\int\left(R(t, y, x, z)-R\left(t, y, x, c_{j}\right)\right) b_{j}(z) d z$.

By (3.5) and Taylor's formula, we have

$$
\begin{aligned}
& \left|R(t, y, x, z)-R\left(t, y, x, c_{j}\right)\right| \\
& \quad \leq C \sum_{k=1}^{n} \sum_{l=1}^{n} \rho\left(z-c_{j}\right)^{a_{k}} \rho(x-y)^{a_{l}} t^{-\gamma-a_{k}-a_{l}}\left(1+t^{-1} \rho\left(y-c_{j}\right)\right)^{-\gamma-1-a_{k}-a_{l}}
\end{aligned}
$$

if $z \in B_{j}, \rho(x-y)<t$ and $y \in \widetilde{B}_{j}^{c}$. Also, we note that

$$
\int \rho(x)^{-\gamma-\alpha+a_{l}}|t-\rho(x)|^{\alpha-1} \chi\left(\frac{\rho(x)}{t}\right) d x=t^{a_{l}-1} \int_{\rho(x)<1} \rho(x)^{-\gamma-\alpha+a_{l}}(1-\rho(x))^{\alpha-1} d x .
$$


SHUICHI SATO

Using these results and Lemma 2.3 (6), we see that

$$
\begin{aligned}
\int_{\mathbb{R}^{n}} & J_{1}^{(3)}(x) d x \\
& \leq C \beta^{2} \sum_{k=1}^{n} \sum_{l=1}^{n} \iint_{\mathbb{R}_{+}^{n+1}} t^{-\gamma-a_{k}-1} \sum_{y \in \widetilde{B}_{j}^{c}}\left|B_{j}\right| r_{j}^{a_{k}}\left(1+t^{-1} \rho\left(y-c_{j}\right)\right)^{-\gamma-1-a_{k}-a_{l}} d y d t \\
& \leq C \beta^{2} \sum_{k=1}^{n} \sum_{l=1}^{n} \sum_{j}\left|B_{j}\right| \iint_{\mathbb{R}_{+}^{n+1}} r_{j}^{a_{k}} t^{-\gamma-a_{k}-1}\left(1+t^{-1}\left(\rho(y)+r_{j}\right)\right)^{-\gamma-1-a_{k}-a_{l}} d y d t .
\end{aligned}
$$

The last integral equals

$$
\iint_{\mathbb{R}_{+}^{n+1}} t^{a_{l}}(1+t)^{-\gamma-1-a_{k}-a_{l}}(\rho(y)+1)^{-\gamma-a_{k}} d y d t
$$

Thus, by Lemma 2.3 (7) with $p=p_{0}$ we have

$$
\int_{\mathbb{R}^{n}} J_{1}^{(3)}(x) d x \leq C \beta^{2-p_{0}}\|f\|_{p_{0}}^{p_{0}}
$$

We next evaluate $J_{2}^{(3)}$. We note that

$$
\left|K_{\rho(x-y)} * I_{\alpha}\left(b_{j}\right)(w)\right| \leq C M\left(I_{\alpha} b_{j}\right)(y), \quad \text { for } w=y, x .
$$

Therefore, if $x \in \Omega_{2}^{c}$ and $p_{0}=2 \gamma /(\gamma+2 \alpha)$, using the Schwarz inequality and Lemma 2.2 (1), we see that

$$
\begin{aligned}
J_{2}^{(3)}(x) & \leq C \int_{\mathbb{R}^{n}}\left|\sum_{j} \chi_{B\left(c_{j}, 2 r_{j}\right)}(y) M\left(I_{\alpha} b_{j}\right)(y)\right|^{2} \rho(y-x)^{-\gamma-2 \alpha} d y \\
& \leq C \sum_{j} \int_{B\left(c_{j}, 2 r_{j}\right)}\left|M\left(I_{\alpha} b_{j}\right)(y)\right|^{2} \rho(y-x)^{-\gamma-2 \alpha} d y \\
& \leq C \sum_{j} \rho\left(x-c_{j}\right)^{-\gamma-2 \alpha} \int_{B\left(c_{j}, 2 r_{j}\right)}\left|M\left(I_{\alpha} b_{j}\right)(y)\right|^{2} d y .
\end{aligned}
$$

Consequently, the $L^{2}$ boundedness of the maximal function $M$, Theorem A and Lemma 2.3 (6) with $p=p_{0}$ imply

$$
J_{2}^{(3)}(x) \leq C \sum_{j} \rho\left(x-c_{j}\right)^{-\gamma-2 \alpha}\left\|b_{j}\right\|_{p_{0}}^{2} \leq C \sum_{j} \rho\left(x-c_{j}\right)^{-\gamma-2 \alpha} \beta^{2}\left|B_{j}\right|^{2 / p_{0}} .
$$

Thus, applying Lemma 2.3 (7) with $p=p_{0}$, we see that

$$
\begin{aligned}
\int_{\Omega_{2}^{c}} J_{2}^{(3)}(x) d x & \leq C \sum_{j} \beta^{2}\left|B_{j}\right|^{2 / p_{0}} \int_{\rho\left(x-c_{j}\right) \geq 4 r_{j}} \rho\left(x-c_{j}\right)^{-\gamma-2 \alpha} \\
& \leq C \sum_{j} \beta^{2}\left|B_{j}\right|^{2 / p_{0}} r_{j}^{-2 \alpha} \leq C \sum_{j} \beta^{2}\left|B_{j}\right| \leq C \beta^{2-p_{0}}\|f\|_{p_{0}}^{p_{0}} .
\end{aligned}
$$

Combining (6.2) and (6.3), we have (4.7). 


\section{Proof of Part (1) of Theorem 1.1 For $p>2$}

Let $A_{1}$ be the weight class of Muckenhoupt consisting of those weights $w$ which satisfy $M(w)(x) \leq C w(x)$ a.e. Applying the methods of [8] we prove the following.

Proposition 7.1. Let $w \in A_{1}$. Suppose $0<\alpha<1$. Then we have

$$
\left\|D_{\alpha}(f)\right\|_{2, w} \leq C\|f\|_{2, w},
$$

where $\|f\|_{2, w}$ is the norm in $L_{w}^{2}$ defined as $\|f\|_{2, w}=\left(\int_{\mathbb{R}^{n}}|f(x)|^{2} w(x) d x\right)^{1 / 2}$.

Let

$$
\widetilde{\rho}_{m}(z)=\int(2 \pi \rho(\xi))^{-\alpha} \widetilde{\Phi}\left(2^{m} \rho(\xi)\right) e^{2 \pi i\langle z, \xi\rangle} d \xi
$$

with $\widetilde{\Phi} \in C_{0}^{\infty}\left(\mathbb{R}_{+}\right)$which is identically 1 on the support of $\Phi$, where $\Phi$ is as in the proof of Lemma 3.4. To prove Proposition 7.1 we need the following.

Lemma 7.2. The estimates

$$
\begin{gathered}
\left|\widetilde{\rho}_{m}(z)\right| \leq C \rho(z)^{\alpha-\gamma}, \\
\left|\partial_{s} \widetilde{\rho}_{m}(z)\right| \leq C \rho(z)^{\alpha-\gamma-a_{s}}, \quad 1 \leq s \leq n,
\end{gathered}
$$

hold true with a positive constant $C$ independent of $m \in \mathbb{Z}$ (the set of integers).

Proof. To prove (7.1) we write

$$
\widetilde{\rho}_{m}(z)=2^{m(\alpha-\gamma)} \int(2 \pi \rho(\xi))^{-\alpha} \widetilde{\Phi}(\rho(\xi)) e^{2 \pi i\left\langle A_{2}-m z, \xi\right\rangle} d \xi .
$$

From this we easily have (17.1) when $\rho(z) \leq 2^{m}$. Suppose $2^{m}<\rho(z)$. Then we can prove (7.1) in the same way as (3.7) by applying integration by parts. The estimates in (7.2) can be shown similarly.

Proof of Proposition 7.1, We may assume that $f \in \mathcal{S}\left(\mathbb{R}^{n}\right)$. We also assume that $\sum_{j=-\infty}^{\infty} \Phi\left(2^{j} \rho(\xi)\right)=1$ for $\xi \in \mathbb{R}^{n} \backslash\{0\}$. Define the operator $\Delta_{j}$ by

$$
\widehat{\Delta_{j}(f)}(\xi)=\Phi\left(2^{j} \rho(\xi)\right) \hat{f}(\xi) \quad \text { for } \quad j \in \mathbb{Z} .
$$

Let

$$
\begin{aligned}
& T_{j}(f)(x) \\
= & \left(\sum_{k=-\infty}^{\infty} \int \chi_{[1,2]}\left(2^{-k} \rho(y)\right)\left|I_{\alpha}\left(\Delta_{j+k} f\right)(x+y)-I_{\alpha}\left(\Delta_{j+k} f\right)(x)\right|^{2} \rho(y)^{-\gamma-2 \alpha} d y\right)^{1 / 2} .
\end{aligned}
$$

Then we have

$$
D_{\alpha}(f)(x) \leq \sum_{j=-\infty}^{\infty} T_{j}(f)(x)
$$

If we put $S_{j}=\left\{2^{-j-1} \leq \rho(\xi) \leq 2^{-j+1}\right\}$, the Plancherel theorem implies

(7.4) $\left\|T_{j}(f)\right\|_{2}^{2}$

$$
\leq C \sum_{k=-\infty}^{\infty} \int \chi_{[1,2]}\left(2^{-k} \rho(y)\right) \rho(y)^{-\gamma-2 \alpha}\left(\int_{S_{j+k}}|\hat{f}(\xi)|^{2} \rho(\xi)^{-2 \alpha}\left|1-e^{2 \pi i\langle y, \xi\rangle}\right|^{2} d \xi\right) d y .
$$


If $2^{k} \leq \rho(y) \leq 2^{k+1}, \xi \in S_{j+k}$ and $j \geq 0$, we see that

$$
\left|1-e^{2 \pi i\langle y, \xi\rangle}\right| \leq 2 \pi \sum_{l=1}^{n}\left|y_{l} \xi_{l}\right| \leq C \sum_{l=1}^{n} \rho(y)^{a_{l}} \rho(\xi)^{a_{l}} \leq C \sum_{l=1}^{n} 2^{-j a_{l}} \leq C 2^{-j} .
$$

Also, $\left|1-e^{2 \pi i\langle y, \xi\rangle}\right| \leq 2$. Therefore by (17.4) we have

$$
\begin{aligned}
& \left\|T_{j}(f)\right\|_{2}^{2} \\
& \leq C \sum_{k=-\infty}^{\infty} C 2^{2 j \alpha} \min \left(1,2^{-2 j}\right) \int_{S_{j+k}}|\hat{f}(\xi)|^{2} d \xi \leq C 2^{2 j \alpha} \min \left(1,2^{-2 j}\right)\|f\|_{2}^{2},
\end{aligned}
$$

where the last inequality follows from the bounded overlap of $\left\{S_{j}\right\}$ and the Plancherel theorem.

Next, when $2^{k} \leq \rho(y) \leq 2^{k+1}$, we decompose

$$
\begin{aligned}
& I_{\alpha}\left(\Delta_{j+k} f\right)(x+y)- I_{\alpha}\left(\Delta_{j+k} f\right)(x) \\
&=\int_{\rho(z) \geq 2^{k+2}}\left(\widetilde{\rho}_{j+k}(z-y)-\widetilde{\rho}_{j+k}(z)\right) \Delta_{j+k} f(x+z) d z \\
&+\int_{\rho(z)<2^{k+2}}\left(\widetilde{\rho}_{j+k}(z-y)-\widetilde{\rho}_{j+k}(z)\right) \Delta_{j+k} f(x+z) d z,
\end{aligned}
$$

where $\widetilde{\rho}_{m}$ is as in Lemma 7.2 ,

If $2^{k} \leq \rho(y) \leq 2^{k+1}$ and $\rho(z) \geq 2^{k+2}$, by (7.2)

$$
\left|\widetilde{\rho}_{j+k}(z-y)-\tilde{\rho}_{j+k}(z)\right| \leq C \sum_{l=1}^{n}\left|y_{l}\right| \rho(z)^{-\gamma+\alpha-a_{l}} \leq C \sum_{l=1}^{n} \rho(y)^{a_{l}} \rho(z)^{-\gamma+\alpha-a_{l}} .
$$

By this and an elementary computation concerning the maximal operator $M$, we see that the first integral on the right hand side of (7.6) is majorized by

$$
C \sum_{l=1}^{n} 2^{k a_{l}} \int_{\rho(z) \geq 2^{k+2}} \rho(z)^{-\gamma+\alpha-a_{l}}\left|\Delta_{j+k} f(x+z)\right| d z \leq C 2^{k \alpha} M\left(\Delta_{j+k} f\right)(x) .
$$

Similarly, if $2^{k} \leq \rho(y) \leq 2^{k+1}$, (7.1) implies that the second integral on the right hand side of (7.6) is bounded by

$$
\begin{gathered}
\int_{\rho(z) \leq 2^{k+3}} \rho(z)^{-\gamma+\alpha}\left|\Delta_{j+k} f(x+y+z)\right| d z+\int_{\rho(z) \leq 2^{k+2}} \rho(z)^{-\gamma+\alpha}\left|\Delta_{j+k} f(x+z)\right| d z \\
\leq C 2^{k \alpha} M\left(\Delta_{j+k} f\right)(x+y)+C 2^{k \alpha} M\left(\Delta_{j+k} f\right)(x) .
\end{gathered}
$$

Using these estimates in (7.6), we see that

$\left|I_{\alpha}\left(\Delta_{j+k} f\right)(x+y)-I_{\alpha}\left(\Delta_{j+k} f\right)(x)\right| \leq C 2^{k \alpha} M\left(\Delta_{j+k} f\right)(x+y)+C 2^{k \alpha} M\left(\Delta_{j+k} f\right)(x)$ when $2^{k} \leq \rho(y) \leq 2^{k+1}$.

Thus

$T_{j}(f)(x)^{2} \leq C \sum_{k=-\infty}^{\infty} \int \chi_{[1,2]}\left(2^{-k} \rho(y)\right)\left|M\left(\Delta_{j+k} f\right)(x+y)+M\left(\Delta_{j+k} f\right)(x)\right|^{2} \rho(y)^{-\gamma} d y$ 
and hence, if $w \in A_{1}$,

$$
\begin{aligned}
\int_{\mathbb{R}^{n}} & T_{j}(f)(x)^{2} w(x) d x \\
& \leq C \sum_{k=-\infty}^{\infty}\left(\int_{\mathbb{R}^{n}}\left|M\left(\Delta_{j+k}\right) f(x)\right|^{2} M(w)(x) d x+\int_{\mathbb{R}^{n}}\left|M\left(\Delta_{j+k} f\right)(x)\right|^{2} w(x) d x\right) \\
& \leq C \sum_{k=-\infty}^{\infty} \int_{\mathbb{R}^{n}}\left|\Delta_{j+k} f(x)\right|^{2} w(x) d x,
\end{aligned}
$$

where the last inequality follows from the defining property of the $A_{1}$ weights and the $L_{w}^{2}$ boundedness of $M$ with $w \in A_{1}$. Thus the Littlewood-Paley inequality in $L_{w}^{2}$ implies

$$
\left\|T_{j}(f)\right\|_{2, w} \leq C\|f\|_{2, w} .
$$

Interpolating between (7.5) and (7.7) with change of measures, and noting that for any $w \in A_{1}$ there is $\delta>0$ such that $w^{1+\delta} \in A_{1}$, we have

$$
\left\|T_{j}(f)\right\|_{2, w} \leq C 2^{-\epsilon|j|}\|f\|_{2, w}
$$

with some $\epsilon>0$ for $w \in A_{1}$, if $0<\alpha<1$. This implies the desired inequality in Proposition 7.1 via (7.3).

Now we can prove part (1) of Theorem 1.1 for $p>2$. Choose a non-negative function $g$ such that $\|g\|_{(p / 2)^{\prime}}=1$ and $\left\|D_{\alpha}(f)\right\|_{p}^{2}=\int\left|D_{\alpha}(f)(x)\right|^{2} g(x) d x$, where $(p / 2)^{\prime}$ denotes the exponent conjugate to $p / 2$. For $s>1$, let $M_{s}(g)=M\left(g^{s}\right)^{1 / s}$. Then $g \leq M_{s}(g)$ a.e. and it is known that $M_{s}(g) \in A_{1}$. Thus by Proposition 7.1 we have

$$
\int\left|D_{\alpha}(f)(x)\right|^{2} g(x) d x \leq \int\left|D_{\alpha}(f)(x)\right|^{2} M_{s}(g)(x) d x \leq C \int|f(x)|^{2} M_{s}(g)(x) d x .
$$

Applying Hölder's inequality to the last integral with $1<s<(p / 2)^{\prime}$, by $L^{(p / 2)^{\prime}}$ boundedness of $M_{s}$ we see that

$$
\int\left|D_{\alpha}(f)(x)\right|^{2} g(x) d x \leq C\|f\|_{p}^{2}\left\|M_{s}(g)\right\|_{(p / 2)^{\prime}} \leq C\|f\|_{p}^{2} .
$$

Combining results, we can get the desired estimate.

\section{REMARKS}

We conclude this note with three remarks.

Remark 8.1. Let $0<\alpha<\gamma$. The Fourier transform of $(2 \pi \rho(\xi))^{-\alpha}$ is a function $\mathcal{R}_{\alpha}(x)$ which is homogeneous of degree $\alpha-\gamma$ with respect to $A_{t}$ and in $C^{\infty}\left(\mathbb{R}^{n} \backslash\{0\}\right)$ (see [3] and [13, Chapter I]). Thus we have

$$
I_{\alpha}(f)(x)=\int_{\mathbb{R}^{n}} \mathcal{R}_{\alpha}(x-z) f(z) d z, \quad f \in \mathcal{S}\left(\mathbb{R}^{n}\right) .
$$

Remark 8.2. Let $0<\alpha<1, p_{0}=2 \gamma /(\gamma+2 \alpha)$ and $p_{0}>1$ as in the hypotheses of Theorem 1.1. Then, if $1 \leq p<p_{0}, D_{\alpha}$ is not of weak type $(p, p)$. Since $D_{\alpha}$ is bounded on $L^{2}\left(\mathbb{R}^{n}\right)$, by taking into account the interpolation of Marcinkiewicz, to show this it suffices to prove that $D_{\alpha}$ is not bounded on $L^{p}\left(\mathbb{R}^{n}\right)$ when $1<p<p_{0}$. 
To see this, we prove that if $D_{\alpha}$ is bounded on $L^{p}\left(\mathbb{R}^{n}\right)$ with $1 \leq p \leq 2$, then $p \geq p_{0}$. Let $A(x)=\left\{y \in \mathbb{R}^{n}: 1 / 2 \leq \rho(y-x) \leq 1\right\}$. Let $\eta$ be a non-zero element in $\mathcal{S}\left(\mathbb{R}^{n}\right)$ with $\operatorname{supp}(\hat{\eta}) \subset\{1 \leq \rho(\xi) \leq 2\}$. Then

$$
\begin{aligned}
D_{\alpha}(\eta)(x) & \geq\left(\int_{A(0)}\left|I_{\alpha}(\eta)(x+y)-I_{\alpha}(\eta)(x)\right|^{2} d y\right)^{1 / 2} \\
& \geq\left(\int_{A(0)}\left|I_{\alpha}(\eta)(x+y)\right|^{2} d y\right)^{1 / 2}-\left(\int_{A(0)}\left|I_{\alpha}(\eta)(x)\right|^{2} d y\right)^{1 / 2} \\
& =\left(\int_{A(0)}\left|I_{\alpha}(\eta)(x+y)\right|^{2} d y\right)^{1 / 2}-|A(0)|^{1 / 2}\left|I_{\alpha}(\eta)(x)\right| .
\end{aligned}
$$

Therefore

$$
\left(\int_{A(x)}\left|I_{\alpha}(\eta)(y)\right|^{2} d y\right)^{1 / 2} \leq D_{\alpha}(\eta)(x)+C\left|I_{\alpha}(\eta)(x)\right|
$$

We have

$$
\left(\int_{\mathbb{R}^{n}}\left|I_{\alpha}(\eta)(y)\right|^{2} d y\right)^{p / 2} \leq C \int_{\mathbb{R}^{n}}\left(\int_{A(x)}\left|I_{\alpha}(\eta)(y)\right|^{2} d y\right)^{p / 2} d x
$$

Let $S(x, r)=\left\{y \in \mathbb{R}^{n}:|x-y|<r\right\}$ for $x \in \mathbb{R}^{n}$ and $r>0$. To see (8.2), we consider a covering of $\mathbb{R}^{n}: \cup_{j=1}^{\infty} A\left(x^{(j)}\right)=\mathbb{R}^{n}$, for all $x^{(j)} \in S\left(c_{j}, \tau\right), j=1,2, \ldots$, where $S\left(c_{j}, \tau\right) \cap S\left(c_{k}, \tau\right)=\emptyset$ if $j \neq k$. Then we see that

$$
\left(\int_{\mathbb{R}^{n}}\left|I_{\alpha}(\eta)(y)\right|^{2} d y\right)^{p / 2} \leq \sum_{j=1}^{\infty}\left(\int_{A\left(x^{(j)}\right)}\left|I_{\alpha}(\eta)(y)\right|^{2} d y\right)^{p / 2}
$$

for all $x^{(j)} \in S\left(c_{j}, \tau\right), j=1,2, \ldots$, since $p / 2 \leq 1$. It follows that

$$
\begin{aligned}
\left(\int_{\mathbb{R}^{n}}\left|I_{\alpha}(\eta)(y)\right|^{2} d y\right)^{p / 2} & \leq \sum_{j=1}^{\infty} \inf _{x^{(j)} \in S\left(c_{j}, \tau\right)}\left(\int_{A\left(x^{(j)}\right)}\left|I_{\alpha}(\eta)(y)\right|^{2} d y\right)^{p / 2} \\
& \leq C_{\tau} \sum_{j=1}^{\infty} \int_{S\left(c_{j}, \tau\right)}\left(\int_{A(x)}\left|I_{\alpha}(\eta)(y)\right|^{2} d y\right)^{p / 2} d x \\
& \leq C_{\tau} \int_{\mathbb{R}^{n}}\left(\int_{A(x)}\left|I_{\alpha}(\eta)(y)\right|^{2} d y\right)^{p / 2} d x
\end{aligned}
$$

which proves (8.2). By (8.1) and (8.2), we have

$$
\left\|I_{\alpha}(\eta)\right\|_{2} \leq C\left\|D_{\alpha}(\eta)\right\|_{p}+C\left\|I_{\alpha}(\eta)\right\|_{p} .
$$

Thus if $\left\|D_{\alpha}(\eta)\right\|_{p} \leq C\|\eta\|_{p}$, we have

$$
\left\|I_{\alpha}(\eta)\right\|_{2} \leq C\|\eta\|_{p}+C\left\|I_{\alpha}(\eta)\right\|_{p} .
$$

Using this with $\eta_{t}$ in place of $\eta$ and homogeneity, we readily see that

$$
t^{\alpha-\gamma / 2} \leq C t^{-\gamma+\gamma / p}+C t^{\alpha+\gamma(1 / p-1)} \leq C t^{-\gamma+\gamma / p}
$$

for all $t \in(0,1)$, which implies that $p \geq 2 \gamma /(\gamma+2 \alpha)$ as claimed. 
Remark 8.3. Define the Littlewood-Paley function

$$
g_{Q}(f)(x)=\left(\int_{0}^{\infty}\left|Q_{t} * f(x)\right|^{2} \frac{d t}{t}\right)^{1 / 2}
$$

where $Q$ is as in (3.2). Then it is known that

$$
c_{1}\|f\|_{p} \leq\left\|g_{Q}(f)\right\|_{p} \leq c_{2}\|f\|_{p}, \quad 1<p<\infty,
$$

with positive constants $c_{1}, c_{2}$ independent of $f$ (see [14]). Also, we can show that

$$
g_{Q}(f)(x) \leq C_{\alpha} D_{\alpha}(f)(x), \quad 0<\alpha<1,
$$

for $f \in \mathcal{S}\left(\mathbb{R}^{n}\right)$, similarly to [22, p. $\left.162,6.12\right]$, which implies the reverse inequality of $\left\|D_{\alpha}(f)\right\|_{p} \leq C\|f\|_{p}$ in part (1) of Theorem 1.1.

Here we give a proof of (8.3) in more details for completeness. Let

$$
U_{\alpha}(x, t)=K_{t} * I_{\alpha}(f)(x)=\int \hat{f}(\xi)(2 \pi \rho(\xi))^{-\alpha} e^{-2 \pi t \rho(\xi)} e^{2 \pi i\langle x, \xi\rangle} d \xi,
$$

where $K$ is as in (1.4). Then

$$
\partial_{0}^{2} U_{\alpha}(x, t)=\int \hat{f}(\xi)(2 \pi \rho(\xi))^{-\alpha+2} e^{-2 \pi t \rho(\xi)} e^{2 \pi i\langle x, \xi\rangle} d \xi
$$

where $\partial_{0}=\partial / \partial t$, and

$$
\begin{aligned}
\int_{0}^{\infty} \partial_{0}^{2} U_{\alpha}(x, t+s) s^{-\alpha} d s & =\left(\int_{0}^{\infty} e^{-s} s^{-\alpha} d s\right) \int \hat{f}(\xi)(2 \pi \rho(\xi)) e^{-2 \pi t \rho(\xi)} e^{2 \pi i\langle x, \xi\rangle} d \xi \\
& =-\Gamma(1-\alpha) \frac{1}{t} Q_{t} * f(x) .
\end{aligned}
$$

Using this, we see that

$$
\begin{aligned}
& \left(\int_{0}^{\infty}\left|Q_{t} * f(x)\right|^{2} \frac{d t}{t}\right)^{1 / 2}=\Gamma(1-\alpha)^{-1}\left(\int_{0}^{\infty} t\left|\int_{0}^{\infty} \partial_{0}^{2} U_{\alpha}(x, t+s) s^{-\alpha} d s\right|^{2} d t\right)^{1 / 2} \\
& =\Gamma(1-\alpha)^{-1}\left(\int_{0}^{\infty}\left|\int_{0}^{\infty} t^{3 / 2-\alpha} \chi_{[1, \infty)}(s)\right| s-\left.\left.1\right|^{-\alpha} \partial_{0}^{2} U_{\alpha}(x, s t) d s\right|^{2} d t\right)^{1 / 2} .
\end{aligned}
$$

By Minkowski's inequality, this is bounded by

$$
\begin{aligned}
& \Gamma(1-\alpha)^{-1} \int_{1}^{\infty}(s-1)^{-\alpha}\left(\int_{0}^{\infty} t^{2(3 / 2-\alpha)}\left|\partial_{0}^{2} U_{\alpha}(x, s t)\right|^{2} d t\right)^{1 / 2} d s \\
& =\Gamma(1-\alpha)^{-1}\left(\int_{1}^{\infty}(s-1)^{-\alpha} s^{-2+\alpha} d s\right)\left(\int_{0}^{\infty} t^{3-2 \alpha}\left|\partial_{0}^{2} U_{\alpha}(x, t)\right|^{2} d t\right)^{1 / 2} .
\end{aligned}
$$

Thus

$$
g_{Q}(f)(x) \leq C_{\alpha}\left(\int_{0}^{\infty} t^{3-2 \alpha}\left|\partial_{0}^{2} U_{\alpha}(x, t)\right|^{2} d t\right)^{1 / 2} .
$$

Since $\int \partial_{0}^{2} K_{t}=0$, we have

$$
\partial_{0}^{2} U_{\alpha}(x, t)=\int \partial_{0}^{2} K_{t}(y) I_{\alpha} f(x+y) d y=\int \partial_{0}^{2} K_{t}(y)\left(I_{\alpha} f(x+y)-I_{\alpha} f(x)\right) d y \text {. }
$$

Arguing similarly to the proof of Lemma 3.1. we see that

$$
\left|\partial_{0}^{2} K_{t}(y)\right| \leq C(t+\rho(y))^{-\gamma-2} .
$$


Using this, we have

$$
\begin{aligned}
& \left|\partial_{0}^{2} U_{\alpha}(x, t)\right| \leq C \int(t+\rho(y))^{-\gamma-2}\left|I_{\alpha} f(x+y)-I_{\alpha} f(x)\right| d y \\
& \leq C \int_{\rho(y)<t} t^{-\gamma-2}\left|I_{\alpha} f(x+z)-I_{\alpha} f(x)\right| d y+C \int_{\rho(y) \geq t} \rho(y)^{-\gamma-2}\left|I_{\alpha} f(x+z)-I_{\alpha} f(x)\right| d y .
\end{aligned}
$$

@@ It follows that

$$
\int_{0}^{\infty} t^{3-2 \alpha}\left|\partial_{0}^{2} U_{\alpha}(x, t)\right|^{2} d t \leq C(I+I I)
$$

where

$$
\begin{gathered}
I=\int_{0}^{\infty} t^{3-2 \alpha}\left(\int_{\rho(y)<t} t^{-\gamma-2}\left|I_{\alpha} f(x+y)-I_{\alpha} f(x)\right| d y\right)^{2} d t \\
I I=\int_{0}^{\infty} t^{3-2 \alpha}\left(\int_{\rho(y) \geq t} \rho(y)^{-\gamma-2}\left|I_{\alpha} f(x+y)-I_{\alpha} f(x)\right| d y\right)^{2} d t .
\end{gathered}
$$

By the Schwarz inequality

$$
\begin{aligned}
I & \leq C \int_{0}^{\infty} t^{3-2 \alpha} t^{-2(\gamma+2)} t^{\gamma} \int_{\rho(y)<t}\left|I_{\alpha} f(x+y)-I_{\alpha} f(x)\right|^{2} d y d t \\
& =C \int\left|I_{\alpha} f(x+y)-I_{\alpha} f(x)\right|^{2}\left(\int_{\rho(y)}^{\infty} t^{-1-\gamma-2 \alpha} d t\right) d y \\
& =C \frac{1}{\gamma+2 \alpha} D_{\alpha}(f)(x)^{2} .
\end{aligned}
$$

Also,

$$
\begin{aligned}
I I & \leq C \int_{0}^{\infty} t^{3-2 \alpha} t^{-2}\left(\int_{\rho(y) \geq t} \rho(y)^{-\gamma-2}\left|I_{\alpha} f(x+y)-I_{\alpha} f(x)\right|^{2} d y\right) d t \\
& =C \int \rho(y)^{-\gamma-2}\left|I_{\alpha} f(x+y)-I_{\alpha} f(x)\right|^{2}\left(\int_{0}^{\rho(y)} t^{1-2 \alpha} d t\right) d y \\
& =C \frac{1}{2-2 \alpha} D_{\alpha}(f)(x)^{2} .
\end{aligned}
$$

Therefore

$$
\left(\int_{0}^{\infty} t^{3-2 \alpha}\left|\partial_{0}^{2} U_{\alpha}(x, t)\right|^{2} d t\right)^{1 / 2} \leq C_{\alpha} D_{\alpha}(f)(x)
$$

Combining this with (8.4), we have (8.3).

\section{REFERENCES}

[1] R. Alabern, J. Mateu and J. Verdera, A new characterization of Sobolev spaces on $\mathbb{R}^{n}$, Math. Ann. 354 (2012), 589-626.

[2] A. P. Calderón and A. Torchinsky, Parabolic maximal functions associated with a distribution, Advances in Math. 16 (1975), 1-64.

[3] A. P. Calderón and A. Torchinsky, Parabolic maximal functions associated with a distribution. II, Advances in Math. 24 (1977), 101-171.

[4] O. N. Capri, On an inequality in the theory of parabolic $H^{p}$ spaces, Revista de la Unión Matemática Argentina 32 (1985), 17-28.

[5] S. Chanillo and R. L. Wheeden, Inequalities for Peano maximal functions and Marcinkiewicz integrals, Duke Math. J. 50 (1983), 573-603. 
[6] R. R. Coifman and G. Weiss, Analyse Harmonique Non-Commutative sur Certains Espaces Homogenes, Lecture Notes in Math. 242, Springer-Verlag, Berlin and New York, 1971.

[7] R. R. Coifman and G. Weiss, Extensions of Hardy spaces and their use in analysis, Bull. Amer. Math. Soc. 83 (1977), 569-645.

[8] J. Duoandikoetxea and J. L. Rubio de Francia, Maximal and singular integral operators via Fourier transform estimates, Invent. Math. 84 (1986), 541-561.

[9] E. B. Fabes and N. Rivière, Singular integrals with mixed homogeneity, Studia Math. 27 (1966), 19-38.

[10] C. Fefferman, Inequalities for strongly singular convolution operators, Acta Math. 124 (1970), 9-36.

[11] P. Hajłasz and Z. Liu, A Marcinkiewicz integral type characterization of the Sobolev space, Publ. Mat. 61 (2017), 83-104.

[12] J. Marcinkiewicz, Sur quelues integrales de type de Dini, Annales de la Société Polonaise 17 (1938), 42-50.

[13] A. Nagel and E. M. Stein, Lectures on Pseudo-Differential Operators, Mathematical Notes 24, Princeton University Press, Princeton, NJ, 1979.

[14] N. Rivière, Singular integrals and multiplier operators, Ark. Mat. 9 (1971), 243-278.

[15] S. Sato, Littlewood-Paley operators and Sobolev spaces, Illinois J. Math. 58 (2014), 1025-1039.

[16] S. Sato, Square functions related to integral of Marcinkiewicz and Sobolev spaces, Linear and Nonlinear Analysis 2 (2016), 237-252 (special issue on ISBFS 2015).

[17] S. Sato, Littlewood-Paley equivalence and homogeneous Fourier multipliers, Integr. Equ. Oper. Theory 87 (2017), 15-44.

[18] S. Sato, Spherical square functions of Marcinkiewicz type with Riesz potentials, Arch. Math. 108 (2017), 415-426.

[19] S. Sato, F. Wang, D. Yang and W. Yuan, Generalized Littlewood-Paley characterizations of fractional Sobolev spaces, Commun. Contemp. Math. (to appear).

[20] C. Segovia and R. L. Wheeden, On the function $g_{\lambda}^{*}$ and the heat equation, Studia Math. 37 (1970), 57-93.

[21] E. M. Stein, The characterization of functions arising as potentials, Bull. Amer. Math. Soc. 67 (1961), 102-104.

[22] E. M. Stein, Singular Integrals and Differentiability Properties of Functions, Princeton Univ. Press, 1970.

[23] E. M. Stein and G. Weiss, Introduction to Fourier Analysis on Euclidean Spaces, Princeton Univ. Press, 1971.

Department of Mathematics, Faculty of Education, Kanazawa University, Kanazawa 920-1192, JAPAN

E-mail address: shuichi@kenroku.kanazawa-u.ac.jp 